\title{
Neither heat pulse, nor multigenerational exposure to a modest increase in water temperature, alters the susceptibility of Guadeloupean Biomphalaria glabrata to Schistosoma mansoni infection.
}

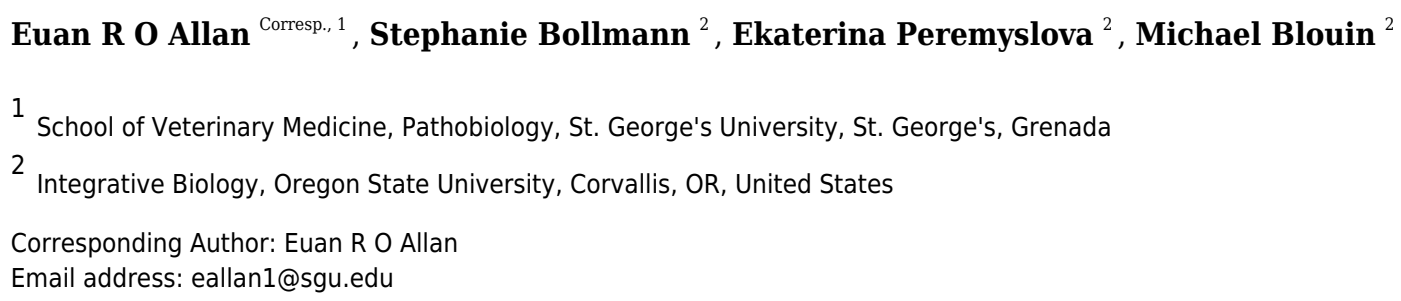

There are increasing concerns regarding the role global climate change will have on many vector-borne diseases. Both mathematical models and laboratory experiments suggest that schistosomiasis risk may change as a result of the effects of increasing temperatures on the planorbid snails that host schistosomes. Heat pulse/heat shock of the BS90 strain of Biomphalaria glabrata was shown to increase the rate of infection by Schistosoma mansoni, but the result was not replicable in a follow up experiment by a different lab. We characterised the susceptibility and cercarial shedding of Guadeloupean B. glabrata after infection with $S$. mansoni under two temperature regimes: multigenerational exposure to small increases in temperature, and extreme heat pulse events. Neither long-term, multigenerational rearing at elevated temperatures, nor transient heat pulse modified the susceptibility of Guadeloupean B. glabrata to infection (prevalence) or shedding of schistosome cercaria (intensity of infection). These findings suggest that heat pulseinduced susceptibility in snail hosts may be dependent on the strain of the snail and/or schistosome, or on some as-yet unidentified environmental co-factor. 
2 Neither heat pulse, nor multigenerational exposure to a modest increase in

3 water temperature, alters the susceptibility of Guadeloupean Biomphalaria

4 glabrata to Schistosoma mansoni infection.

5

6 Euan R. O. Allan ${ }^{1 *}$, Stephanie Bollmann ${ }^{2}$, Ekaterina Peremyslova ${ }^{2}$, and Michael S. Blouin ${ }^{2}$

7

$8{ }^{1}$ School of Veterinary Medicine, Pathobiology, St. George's University, St. George’s, Grenada

10 Integrative Biology, Oregon State University, Corvallis, OR, United States

11

12

$13 *$ Corresponding Author

14 Email: euanroallan@gmail.com (EROA)

15 Department of Pathobiology, School of Veterinary Medicine, St. George’s University, Grenada,

16 West Indies

17

18

19 


\section{Abstract}

There are increasing concerns regarding the role global climate change will have on

many vector-borne diseases. Both mathematical models and laboratory experiments suggest that

23 schistosomiasis risk may change as a result of the effects of increasing temperatures on the

24 planorbid snails that host schistosomes. Heat pulse/heat shock of the BS90 strain of

Biomphalaria glabrata was shown to increase the rate of infection by Schistosoma mansoni, but

26 the result was not replicable in a follow up experiment by a different lab. We characterised the

27 susceptibility and cercarial shedding of Guadeloupean B. glabrata after infection with $S$.

28 mansoni under two temperature regimes: multigenerational exposure to small increases in

29 temperature, and extreme heat pulse events. Neither long-term, multigenerational rearing at

30 elevated temperatures, nor transient heat pulse modified the susceptibility of Guadeloupean $B$.

31 glabrata to infection (prevalence) or shedding of schistosome cercaria (intensity of infection).

32 These findings suggest that heat pulse-induced susceptibility in snail hosts may be dependent on

33 the strain of the snail and/or schistosome, or on some as-yet unidentified environmental co-

34 factor. 
38

39

40

41

42

\section{Introduction}

Parasitic trematodes can cause numerous mammalian diseases. The most notable and detrimental human disease, schistosomiasis, is caused by the blood fluke Schistosoma mansoni.

Schistosomiasis is estimated to affect hundreds of millions of people a year, exert massive health and economic tolls on tropical countries, and contribute to hundreds of thousands of deaths $[1$, 2]. Freshwater snails of the genus Biomphalaria serve as obligate intermediate hosts for $S$. mansoni (Sm). Biomphalaria glabrata $(\mathrm{Bg})$ is the most important host for $S$. mansoni in the new world. Schistosome eggs from infected human host feces release miracidia, which penetrate and infect snails. The parasites transform and develop in these snails over a few weeks to become cercariae, which are shed from the snail and go on to infect human hosts and cause disease. It has been postulated that increasing average temperatures, as a result of global climate change, will affect the distribution, population sizes, fecundity, survival, and transmission dynamics of many species involved in vector-borne diseases, including Biomphalaria sp. [3-5].

In the context of snail infection, both parasite and host are fully exposed to external abiotic factors. Miracidia are free swimming and actively infect ectothermic snails in aquatic ecosystems. As such, the physiology and immunity of Bg has been shown to be modified by transient changes in water temperature [6-12]. Models of long-term increases in water temperate in natural ecosystems suggest that Biomphalaria populations and infection dynamics may lead to increases in human and snail infection risk [4]. Concurrently, heat wave exposure is postulated to increase as a result of global climate change as natural fluctuations in temperature are exacerbated [13]. Heat waves can result in spikes in water temperature and heat shock of ectotherms. 

susceptibility of this strain to schistosome infection, and the effect appeared to be mediated by

62

expression of heat shock proteins [6-8]. On the other hand, a follow up study in a different lab failed to replicate the increase in susceptibility in BS90 after heat pulse, a result which suggests that there may be specific effects of the strain of BS90 or Sm used, or that some unidentified environmental co-factor is involved $[9,10]$.

Models of the effects of climate change on schistosomiasis risk could be improved by understanding any changes in transmission rate that result from temperature change per se. Therefore, it seems important to determine how generalizable the effect of heat pulse is in other populations of $\mathrm{Bg}$ and $\mathrm{Sm}$. We set out to determine if a long-term subtle increase in temperature or a transient heat pulse (both ecologically relevant with global climate change), can alter the infection dynamics of another snail-schistosome pair: Guadeloupean Bg (BgGUA) challenged with Guadeloupean Sm (SmGUA).

To examine the effects of a modest but consistent increase in ambient temperature, we maintained BgGUA at $1^{\circ} \mathrm{C}$ above their standard conditions for 7 months $\left(>3\right.$ generations at $27^{\circ} \mathrm{C}$ vs the standard $25-26^{\circ} \mathrm{C}$ ). Though there are huge variations in the estimate of increases to the average temperature in tropical freshwater aquatic ecosystems over the past few decades, we believe that $1^{\circ} \mathrm{C}\left(27^{\circ} \mathrm{C}\right)$ is a reasonable conservative estimate $[3,14,15]$. Additionally, mathematical models suggest that Biophalaria populations may begin to crash when in natural aquatic ecosystems above a consistent water temperature of $28^{\circ} \mathrm{C}$ [4]. In a separate experiment, we transiently heat pulsed $\left(32^{\circ} \mathrm{C}\right) \mathrm{BgGUA}$ for $6 \mathrm{~h}$ before challenge to determine if their susceptibility to SmGUA was altered by heat pulse (and likely heat shock) responses. Given the heterogeneity of heat pulse infection phenotypes and adaptability of schistosomes to numerous 
83 geographical ecosystems, we hypothesised that infection of BgGUA with SmGUA would not be

84 altered by changes in water temperature. We found that neither long-term multigenerational

85 rearing at elevated temperatures nor transient heat pulse modified BgGUA's susceptibly to

86 challenge by SmGUA. These findings suggest the effects of heat on susceptibility of Bg to Sm

87 may be constrained to specific circumstances. 
88

89

90

91

92

93

94

95

96

97

98

99

100

101

102

103

104

105

106

107

108

109

110

\section{Materials and Methods}

\subsection{Biomphalaria glabrata maintenance and ethics.}

Snails (BgGUA) were collected from Guadeloupe in 2005 and maintained as previously described unless otherwise stated [16-23]. All snails were kept in dechlorinated water. Heat pulses conducted using incubators, with constant water temperature monitoring, and long-term small temperature modifications were conducted using thermal stratification in a single room with hourly followed by daily water temperature monitoring. All experiments followed the Public Health Service Domestic Assurance for humane care and use of laboratory animals (PHS Animal Welfare Assurance Number A3229-01), as Animal Care and Use Proposal 4360; approved by Oregon State University Institutional Animal Care and Use Committee.

\subsection{BgGUA long-term temperature exposure and infection studies.}

To examine the effects of a modest but long-term temperature increases, BgGUA were maintained in $25^{\circ} \mathrm{C}$ (standard for some other strains e.g. BS-90), $26^{\circ} \mathrm{C}$ (standard for $\mathrm{BgGUA}$ ), or $27^{\circ} \mathrm{C}$ (elevated) for $>7$ months (all temperatures monitored daily). Adult snails $(>12 \mathrm{~mm})$ were allowed to mate, lay eggs, and be in the presence of the juveniles until the juveniles reached $>3 \mathrm{~mm}$; after which all juveniles were re-tanked (thus eliminating the inclusion of egg sacs from previous generations) and the previous generation was sacrificed. This was done 3 times to ensure $>3$ generations were maintained at a specific temperature. Schistosome challenges were carried out as previously described with some modifications $[6,10,19,20]$. In brief, size matched $(\sim 7 \mathrm{~mm})$ BgGUA were individually challenged with 10 miracidia for $12 \mathrm{~h}$ in $2 \mathrm{ml}$ of dechlorinated water, and transferred into tubs containing up to 10 snails each $(n=41,48,46$ for 
$11125^{\circ} \mathrm{C}, 26^{\circ} \mathrm{C}$, or $27^{\circ} \mathrm{C}$ respectively). All challenges, and maintenance post-challenge, was

112 conducted in water corresponding to the experimental maintenance temperature $\left(25^{\circ} \mathrm{C}, 26^{\circ} \mathrm{C}\right.$, or

$11327^{\circ} \mathrm{C}$ ). Weekly (from week 5 to week 10), snails were placed under light for $3 \mathrm{~h}$ in 24 well dishes

114 in $2 \mathrm{ml}$ of dechlorinated water (at $25^{\circ} \mathrm{C}, 26^{\circ} \mathrm{C}$, or $27^{\circ} \mathrm{C}$ ) and examined for cercarial shedding.

115 They were scored as infected or uninfected, the number of cercaria shed per infected snail was

116 counted, and non-shedding snails were returned to the tank for future assessment $(n=18,18,21$

117 for $25^{\circ} \mathrm{C}, 26^{\circ} \mathrm{C}$, or $27^{\circ} \mathrm{C}$ respectively). All proportions are cumulative for the 10 week period, and

118 cercarial counts are from the first shedding incidence before infected snails were sacrificed.

119

120

\subsection{BgGUA heat pulse and infection studies.}

121

To assess the effects of heat pulse, size matched $(\sim 7 \mathrm{~mm})$ BgGUA were removed from standard conditions $\left(26^{\circ} \mathrm{C}\right.$ ) and exposed to $26^{\circ} \mathrm{C}$ (control), or $32^{\circ} \mathrm{C}$ (heat pulse) for $6 \mathrm{~h}$ prior to challenge with miracidia. After removal from temperature exposure, snails were immediately challenged with 10 miracidia at $26^{\circ} \mathrm{C}$ to ensure no effects of temperature on schistosome activity. Challenges were carried out as previously described [19, 20]. BgGUA were individually challenged for $12 \mathrm{~h}$ in $2 \mathrm{ml}$ of dechlorinated water, transferred into tubs containing up to 10 snails each, and monitored for 10 weeks. Weekly (from week 5 to week 10), snails were placed under light for $3 \mathrm{~h}$ in 24 well dishes in $2 \mathrm{ml}$ of dechlorinated water and examined for cercarial shedding, scored as infected or uninfected ( $n=48$ and 49 for $26^{\circ} \mathrm{C}$, or $32^{\circ} \mathrm{C}$ respectively), and the number of cercaria shed per snail was counted ( $n=15$ for both treatments), and non-shedding snails were returned to the tank for future assessment. All proportions are cumulative for the 10 week period, and cercarial counts are from the first shedding incidence before infected snails were sacrificed. 


\section{$135 \quad 2.4$ Statistical analyses.}

136 Statistical analyses on the number of cercaria shed were completed by one-way ANOVA

137 (or unpaired Student's $t$-test) with a Tukey-test, while analysis of the proportion infected was

138 done by calculating the $\mathrm{Z}$ score (standard score) of the population $(p<0.05)$ [20]. Analyses were

139 completed using GraphPad Prism software (La Jolla, CA, USA).

140

141

142 


\section{3. Results}

145 3.1 BgGUA maintained at $1^{\circ} \mathrm{C}$ above standard conditions for multiple generations, or transiently heat pulsed $6^{\circ} \mathrm{C}$ above standard conditions, do not show altered susceptibility to SmGUA. of a given snail to resist infection, and has relevance for the number of disease transmitting snail hosts. Snail burden of infection, measured by cercarial count during first shedding (only shedding event quantified), was recorded as a measure of transmission risk by each snail. BgGUA, regardless of maintenance temperature $\left(25^{\circ} \mathrm{C}, 26^{\circ} \mathrm{C}\right.$, or $\left.27^{\circ} \mathrm{C}\right)$, had equivalent susceptibility and cercarial shedding when exposed to SmGUA (Figure 1A and B). Additionally, heat pulse $\left(32^{\circ} \mathrm{C}\right)$ did not alter the susceptibility or the number of cercaria shed by BgGUA (Figure 2A and B). Most snails shed cercaria by week 6-7 regardless of treatment (Raw data file). No snail mortality was observed, though infected snails were sacrificed after shedding and could have feasibly died from the infection if they were returned to the population rather than sacrificed. 
161

162

163

164

165

166

167

168

169

170

171

172

173

174

175

176

177

178

179

180

181

182

183

\section{Discussion}

As the potential impacts of global climate change on vector-borne diseases become more evident, and climate change worsens, the importance of understanding these ramifications are accentuated. Our findings support the hypothesis that neither permanent nor transient increases in temperature alter BgGUA susceptibility to infection by SmGUA. Though we are the first to examine prolonged temperature increases in BgGUA, it has been previously discussed that subtle changes in temperature do not generally alter infection dynamics in some other snail-schistosome combinations [6]. Given the strain specific nature of schistosome infectivity, it was important to determine if these long-term temperature changes could modify this strain's susceptibility. Our findings confirm that long-term/subtle elevations in temperature, as a result of climate change, may not modify snail resistance to schistosome challenge. They also speak to the robustness of snail-schistosome infectivity dynamics. Additionally, the burden of SmGUA infection, measured by counting cercarial shedding, was equivalent under all temperature conditions, and therefore temperature fluctuations are not likely to alter schistosome transmission risk from individual BgGUA. These findings also support the notion that the effects of heat pulse/heat shock on schistosome resistance are not general, and are probably strain specific or dependent on particular environmental conditions $[9,10]$.

Specifically, it is important to note that BgGUA strain is less resistant to schistosomes than the strain used in other heat pulse experiments, the BS90 strain. Therefore, our study provides insights into the effects of heat treatments on more susceptible strains of snails, which we believe weakens the conclusion that heat waves could increase infectivity in nature. It is feasible that snails which are already susceptible to schistosome challenge would be even more vulnerable to abiotic effects increasing susceptibility, but we do not observe this. We also 
184 believe the conclusion that heat shock increases snail susceptibility could be further confounded 185 by the possible effects of abiotic components like water quality, humidity, diet, and infection conditions. These subtle variations in the environment could disrupt the effects of temperature, thus weakening or masking the overall importance of heat pulse on schistosome infectivity.

\section{Conclusions}

Although it does not appear that an increase in susceptibility to Sm following heat pulse is a general result, it is important to remember that temperature and climate can affect many biotic factors (including fecundity, growth rates, mortality, and mobility) that influence the transmission of Sm. Controlled laboratory infections are not necessarily representative of what will occur in nature. For example, it is possible that a natural population of $\mathrm{Bg}$ could become more susceptible to $\mathrm{Sm}$, but that they have reduced fecundity resulting in the transmission of fewer parasites [3]. Permanent climatic changes could also shift the ranges of these species because they can only tolerate a finite increase in temperature $[4,5]$. These interacting factors could create different, but not necessarily larger, regions of high schistosomiasis risk. As such, it is important to determine how geographically distinct $\mathrm{Bg}$ and $\mathrm{Sm}$ respond to changes in abiotic factors. Determining the direct effects of these changing environmental conditions on schistosome infection and risk, in a local context, will allow for more accurate schistosomiasis risk models.

\section{Acknowledgements}

This work was supported by the National Institutes of Health [AI109134]. 
207

208

209

210

211

212

213

214

215

216

217

218

219

220

221

222

223

224

225

226

227

228

229

230

231

232

233

234

235

236

237

238

239

240

241

242

243

244

245

246

247

248

249

250

\section{References:}

[1] Schistosomiasis: population requiring preventive chemotherapy and number of people treated in 2010, Wkly Epidemiol Rec 87(4) (2012) 37-44.

[2] Schistosomiasis: number of people treated worldwide in 2014, Wkly Epidemiol Rec 91(5) (2016) 5360.

[3] N. McCreesh, M. Arinaitwe, W. Arineitwe, E.M. Tukahebwa, M. Booth, Effect of water temperature and population density on the population dynamics of Schistosoma mansoni intermediate host snails, Parasit Vectors 7 (2014) 503.

[4] N. McCreesh, M. Booth, The effect of increasing water temperatures on Schistosoma mansoni transmission and Biomphalaria pfeifferi population dynamics: an agent-based modelling study, PLoS One 9(7) (2014) e101462.

[5] N. McCreesh, G. Nikulin, M. Booth, Predicting the effects of climate change on Schistosoma mansoni transmission in eastern Africa, Parasit Vectors 8 (2015) 4.

[6] W. Ittiprasert, M. Knight, Reversing the Resistance Phenotype of the Biomphalaria glabrata Snail Host Schistosoma mansoni Infection by Temperature Modulation, Plos Pathog 8(4) (2012).

[7] M. Knight, O. Elhelu, M. Smith, B. Haugen, A. Miller, N. Raghavan, C. Wellman, C. Cousin, F. Dixon, V. Mann, G. Rinaldi, W. Ittiprasert, P.J. Brindley, Susceptibility of Snails to Infection with Schistosomes is influenced by Temperature and Expression of Heat Shock Proteins, Epidemiology (Sunnyvale) 5(2) (2015).

[8] M. Knight, W. Ittiprasert, H.D. Arican-Goktas, J.M. Bridger, Epigenetic modulation, stress and plasticity in susceptibility of the snail host, Biomphalaria glabrata, to Schistosoma mansoni infection, Int J Parasitol 46(7) (2016) 389-94.

[9] M.K. Nelson, B.C. Cruz, K.L. Buena, H. Nguyen, J.T. Sullivan, Effects of abnormal temperature and starvation on the internal defense system of the schistosome-transmitting snail Biomphalaria glabrata, J Invertebr Pathol 138 (2016) 18-23.

[10] J. Sullivan, Reversal of schistosome resistance in Biomphalaria glabrata by heat shock may be dependent on snail genotype, J Parasitol (2018).

[11] J.R. Coelho, F.S. Bezerra, The effects of temperature change on the infection rate of Biomphalaria glabrata with Schistosoma mansoni, Mem Inst Oswaldo Cruz 101(2) (2006) 223-4.

[12] R.C. Augusto, D. Duval, C. Grunau, Effects of the Environment on Developmental Plasticity and Infection Success of Schistosoma Parasites - An Epigenetic Perspective, Front Microbiol 10 (2019) 1475. [13] R.D. Peng, J.F. Bobb, C. Tebaldi, L. McDaniel, M.L. Bell, F. Dominici, Toward a quantitative estimate of future heat wave mortality under global climate change, Environ Health Perspect 119(5) (2011) 701-6. [14] J.F. Pekel, A. Cottam, N. Gorelick, A.S. Belward, High-resolution mapping of global surface water and its long-term changes, Nature 540(7633) (2016) 418-422.

[15] S. Engels, A.S. Medeiros, Y. Axford, S.J. Brooks, O. Heiri, T.P. Luoto, L. Nazarova, D.F. Porinchu, R. Quinlan, A.E. Self, Temperature change as a driver of spatial patterns and long-term trends in chironomid (Insecta: Diptera) diversity, Glob Chang Biol (2019).

[16] A. Theron, C. Coustau, A. Rognon, S. Gourbiere, M.S. Blouin, Effects of laboratory culture on compatibility between snails and schistosomes, Parasitology 135(10) (2008) 1179-88.

[17] A. Theron, A. Rognon, B. Gourbal, G. Mitta, Multi-parasite host susceptibility and multi-host parasite infectivity: a new approach of the Biomphalaria glabrata/Schistosoma mansoni compatibility polymorphism, Infect Genet Evol 26 (2014) 80-8. 
251 [18] E.R.O. Allan, M.S. Blouin, The behavioral effects of antibiotic treatment on the snail Biomphalaria 252 glabrata, PeerJ 5 (2017) e4171.

253 [19] E.R.O. Allan, B. Gourbal, C.B. Dores, A. Portet, C.J. Bayne, M.S. Blouin, Clearance of schistosome 254 parasites by resistant genotypes at a single genomic region in Biomphalaria glabrata snails involves 255 cellular components of the hemolymph, Int J Parasitol (2017).

256 [20] E.R. Allan, J.A. Tennessen, S.R. Bollmann, P.C. Hanington, C.J. Bayne, M.S. Blouin, Schistosome

257 infectivity in the snail, Biomphalaria glabrata, is partially dependent on the expression of Grctm6, a

258 Guadeloupe Resistance Complex protein, PLoS Negl Trop Dis 11(2) (2017) e0005362.

259 [21] E.R.O. Allan, M.S. Blouin, Allelic variation partially regulates galactose-dependent hydrogen

260 peroxide release from circulating hemocytes of the snail Biomphalaria glabrata, Fish Shellfish Immunol

$26172(2018) 111-116$.

262 [22] E.R.O. Allan, L. Yang, J.A. Tennessen, M.S. Blouin, Allelic variation in a single genomic region alters 263 the hemolymph proteome in the snail Biomphalaria glabrata, Fish Shellfish Immunol 88 (2019) 301-307. 264 [23] E.R.O. Allan, J.A. Tennessen, T.J. Sharpton, M.S. Blouin, Allelic Variation in a Single Genomic Region 265 Alters the Microbiome of the Snail Biomphalaria glabrata, J Hered 109(5) (2018) 604-609.

266

267

268 
Figure 1

Susceptibility and cercarial shedding of BgGUA is not altered by multigenerational maintenance at elevated temperature.

(A) Susceptibility of BgGUA maintained for $>7$ months at $25^{\circ} \mathrm{C}, 26^{\circ} \mathrm{C}$, or $27^{\circ} \mathrm{C}$. Data are presented as proportion of infected snails $+/$ - the standard error of proportions $(n=41,48,46$ for $\left.25^{\circ} \mathrm{C}, 26^{\circ} \mathrm{C}, 27^{\circ} \mathrm{C}\right)$. (B) The total number of cercariae released over $3 \mathrm{~h}$ single shedding event by infected snails. Data are presented as mean $+/-\mathrm{SD}\left(n=18,18,21\right.$ for $25^{\circ} \mathrm{C}, 26^{\circ} \mathrm{C}$, $27^{\circ} \mathrm{C}$ ). No Significant differences ( $Z$ score of proportion; ANOVA $p>0.05$ ).

A

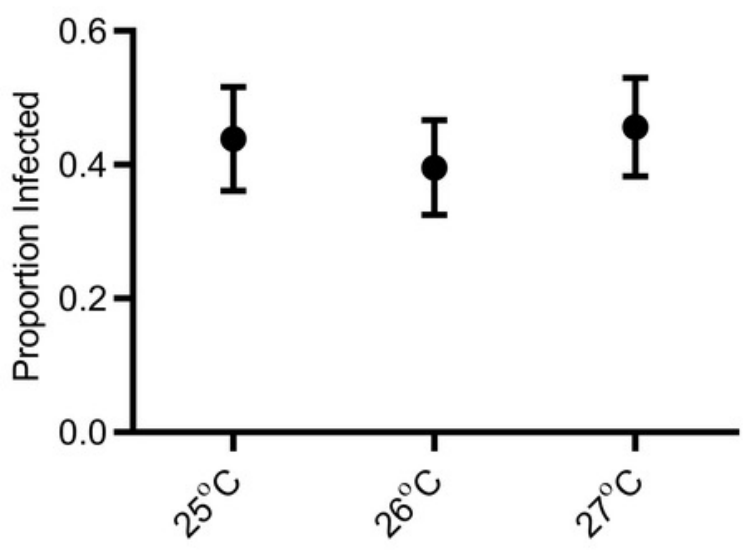

Temperature
B

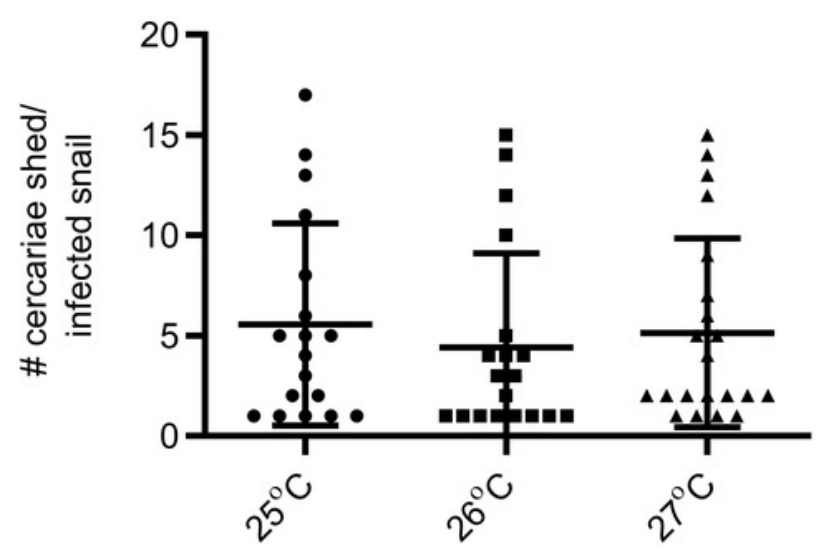

Temperature 
Figure 2

Susceptibility and cercarial shedding of BgGUA is not altered by $6 \mathrm{~h}$ heat pulse prior to schistosome challenge.

(A) Susceptibility of BgGUA pulsed for $6 \mathrm{~h}$ at $26^{\circ} \mathrm{C}$ or $32^{\circ} \mathrm{C}$ (heat pulsed) immediately preceding schistosome challenge. Data are presented as proportion of infected snails $+/$ - the standard error of proportions ( $n=48,49$ for $26^{\circ} \mathrm{C}, 32^{\circ} \mathrm{C}$ ). (B) The total number of cercariae released over $3 \mathrm{~h}$ single shedding event by infected snails. Data are presented as mean $+/$ SD $(n=15)$. No Significant differences ( $Z$ score of proportion; Student $t$-test $p>0.05$ ).

A

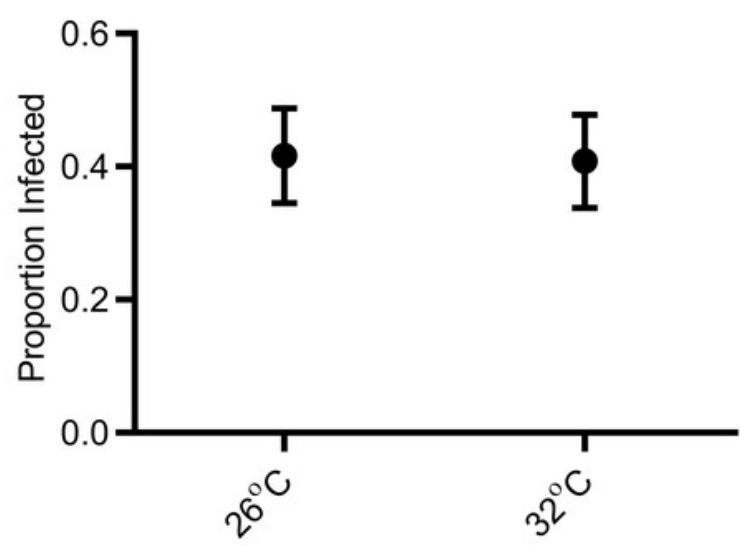

Temperature
B

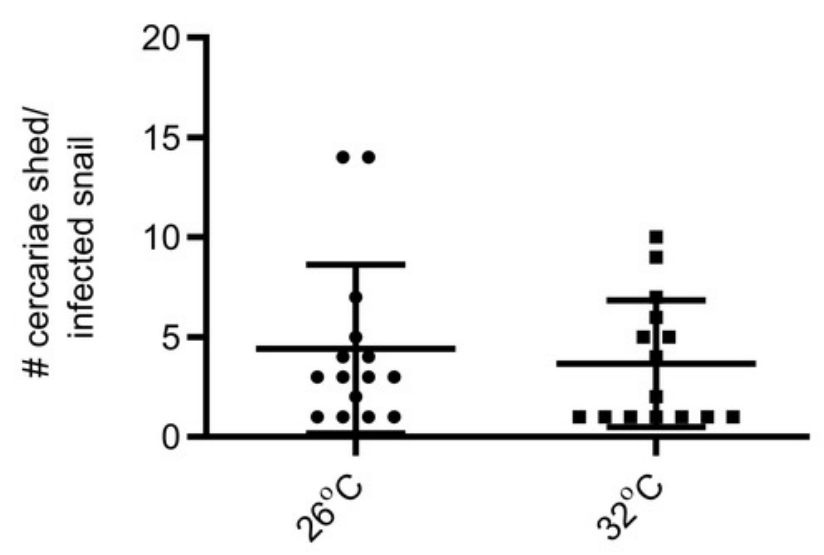

Temperature 